\title{
A novel high-content screening approach for the elucidation of $C$. jejuni biofilm composition and integrity
}

\author{
Matthew V. X. Whelan', Jeremy C. Simpson² and Tadhg Ó Cróinín ${ }^{1 *}$
}

\begin{abstract}
Background: Campylobacter jejuni is the leading cause of bacterial gastroenteritis worldwide and the main source of infection is contaminated chicken meat. Although this important human pathogen is an obligate microaerophile, it must survive atmospheric oxygen conditions to allow transmission from contaminated chicken meat to humans. It is becoming increasingly evident that formation of biofilm plays a key role in the survival of this organism for extended periods on poultry products. We have recently demonstrated a novel inducible model for the study of adherent C. jejuni biofilm formation under aerobic conditions. By taking advantage of supercoiling mediated gene regulation, incubation of C. jejuni with subinhibitory concentrations of the Gyrase B inhibitor novobiocin was shown to promote the consistent formation of metabolically active adherent biofilm.

Results: In this study, we implement this model in conjunction with the fluorescent markers: TAMRA (live cells) and SytoX (dead cells, eDNA) to develop a novel systematic high-content imaging approach and describe how it can be implemented to gain quantifiable information about the integrity and extracellular polymeric substance (EPS) composition of adherent C. jejuni biofilm in aerobic conditions. We show that this produces a model with a consistent, homogenous biofilm that can be induced and used to screen a range of inhibitors of biofilm adherence and matrix formation.
\end{abstract}

Conclusions: This model allows for the first time a high throughput analysis of C. jejuni biofilms which will be invaluable in enabling researchers to develop mechanisms to disrupt these biofilms and reduce the viability of these bacteria under aerobic conditions.

\section{Introduction}

Campylobacter jejuni is the leading cause of bacterial gastroenteritis and a significant health burden across the world, with over 550 million cases reported each year. It is becoming increasingly evident that formation of biofilm is a key aspect of $C$. jejuni virulence, in particular as a stress response mechanism to explain the conundrum of the survival of this highly fastidious organism within the food chain, from farm to fork [1]. Despite this, there

\footnotetext{
* Correspondence: Tadhg.OCroinin@ucd.ie

${ }^{1}$ School of Biomolecular and Biomedical Science, University College Dublin, Belfield, Dublin 4, Ireland

Full list of author information is available at the end of the article
}

remains much to be discovered about the regulation and composition of biofilm formation in C. jejuni. Biofilm formation is influenced by a variety of environmental factors including oxygen availability, temperature, osmolarity and availability of nutrients $[2,3]$. C. jejuni is capable of forming three types of biofilm, (i) a structure adherent to an abiotic surface, (ii) self/mixed-aggregates floating within a liquid culture or (iii) a pellicle layer at the liquid air interface [4]. C. jejuni benefits from enhanced fitness by formation of adherent biofilms in the presence of stressful environmental conditions.

Campylobacter biofilm formation has been shown to be influenced by a broad range of factors including the 
stringent stress response (spoT, Ppk1, Ppk2) [5-7], molecular chaperones (EF-G, ClpP/A/X) [8], twocomponent regulatory systems (CprRS) [9], LOS structure and modification (waaf, lgtF, EptC) [10, 11], OMP profile (Peb4) [12], quorum sensing (luxS) [2], chemotaxis (FucP) and the oxidative stress response (PerR, CosR, CsrA) [8, 13]. Campylobacter adhesins (CadF) and moonlighting proteins (Dps) have been shown to be required for initiation of biofilm formation on an abiotic surface $[14,15]$. Flagellar integrity and motility play key roles in adherence to abiotic surfaces, during chicken colonisation, biofilm formation on human ileal tissue and formation of bacterial cell-cell connections facilitating net-like structures during biofilm maturation $[8,16-$ 18]. Biofilm formation can also be induced by host and environmental factors such as bile salt sodium deoxycholate (DOC), which has been well documented for its role in increasing virulence factor expression, secretion and infection kinetics in C. jejuni $[9,19]$. There is evidence suggesting that induction of $C$. jejuni biofilm on chicken meat may facilitate survival [20]. C. jejuni strain NCTC11168 develops adherent biofilms more rapidly when incubated aerobically rather than under ideal microaerobic gas conditions [3]. Recent work has continued to implicate the role of the oxidative stress response regulon in modulation of biofilm formation when $C$. jejuni is exposed to a high oxygen environment $[21,22]$.

The extracellular polymeric substance (EPS) is a vital component of bacterial biofilms, accounting for as much as $90 \%$ of the biofilm mass [23]. The self-produced extracellular matrix assists the bacteria by providing structural support, antimicrobial resistance, sequestering of nutrients and preventing dehydration [24]. Initially an extracellular fiber-like material in the biofilm produced by NCTC11168 was reported, structurally resembling a net like matrix [8]. Sensitivity of $C$. jejuni biofilm to DNase I treatment revealed extracellular DNA (eDNA) as the likely major constituent in the extracellular matrix (ECM) of adherent C. jejuni biofilms [1, 24]. eDNA release is reported to be independent of functional flagella, insufficient alone to allow for biofilm formation (requiring flagellar mediated adherence) and likely released due to an as yet undefined autolytic mechanism, which has been described in other bacteria such as $P$. aeruginosa $[1,8,9,25]$. A composition assay carried out on the biofilm of six strains found that $1 \mathrm{mg} / \mathrm{mL}$ proteinase $\mathrm{K}$ (protein degradation) promoted almost total degradation of established biofilm, while treatment with $10 \mathrm{mM}$ sodium metaperiodate (carbohydrate oxidation) had little to no effect on biofilm structure [26]. Interestingly, in this same study, three antimicrobial agents used to treat chicken during production, namely paracetic acid $(0.8 \%)$, sodium hyperchlorite $(1 \%)$ and chlorohexidine $(1 \%)$ were screened for their ability to eliminate adherent biofilm of 30 C. jejuni strains, and although they were effective in some cases, $30 \%$ of strains presented with resistance to at least one of the chemical agents while residing within adherent biofilm.

The overarching mechanisms of $C$. jejuni biofilm formation remain elusive. This has been confounded by the relatively low levels of biofilm formed, the lack of information on the viability of the biofilm and the relative low throughput of conventional confocal microscopy in combination with viability staining of biofilms. The lack of sensitivity of conventional assays such as crystal violet staining combined with the extreme heterogeneity of phenotypes observed amongst $C$. jejuni strains has also been a stumbling block. A significant issue in the field has been the lack of a rapid, highly sensitive, systematic approach to screen Campylobacter biofilm formation. Using a fluorescence microscopy approach, we recently demonstrated that relaxation of negative DNA supercoiling promoted an adherent viable biofilm forming phenotype under aeration [27]. Using a combination of microscopy and viability staining with TAMRA, a rhodamine-based dye which is cell permeant and upon entering the cytosol is enzymatically crosslinked by bacterial esterases, preventing the dye from escaping the intact bacterial cell, providing a bright indicator of living bacteria, we were able to detect adherent biofilm phenotypes which were not distinguishable using the crystal violet assay $[28,29]$. The observed induced biofilm forming phenotype correlated with increased invasion of intestinal epithelial cells and killing efficiency of Galleria mellonella larvae for the strain NCTC11168 [27, 30]. This work revealed the potential of using novel microscopy approaches in conjunction with fluorescent viability stains to detect the presence and fluctuations of $C$. jejuni biofilms forming at levels which were not easily resolvable by conventional means.

The aim of this study was to adapt high-content screening approaches to quantitatively measure the effect of a variety of inhibitors of EPS integrity on the novobiocin-induced NCTC11168 biofilm under aeration. The challenge was to design a robust and quantitative approach to systematically analyse the structure and composition of the highly variable Campylobacter biofilm. Traditional microscopy approaches to study Campylobacter biofilm suffer from the limitations of low throughput and subjectivity of image acquisition. In this study, we used high-content screening microscopy in conjunction with TAMRA (live cell) and SytoX (dead cells, eDNA) biofilm labelling to systematically analyse the effect of a broad range of biofilm inhibitors on an induced aerobic biofilm phenotype of strain NCTC11168. Our experimental approach allows for the rapid quantification of the effect of a broad range of inhibitors on 
biofilm viability and structural integrity. This highcontent screening approach to biofilm screening offers a broad range of applications, from rapid characterisation of isolates to screening potential antimicrobial and bactericidal chemical candidates to tackle the growing concern of medically relevant bacterial biofilms.

\section{Materials and methods}

\section{Bacterial strains and growth conditions}

C. jejuni NCTC11168 is the type strain of C. jejuni and a commonly used laboratory strain. $C$. jejuni were cultured on Mueller Hinton (MH) agar (Oxoid) at $37^{\circ} \mathrm{C}$ under microaerobic conditions generated using Campygen gas packs (Oxoid). For liquid cultures, $C$. jejuni strains were equalised to specific optical densities in $\mathrm{MH}$ broth and incubated under microaerobic conditions at $37^{\circ} \mathrm{C}$, shaking at $200 \mathrm{rpm}$. C. jejuni stock cultures were maintained using $\mathrm{MH}$ broth (Oxoid) supplemented with $20 \%$ glycerol and stored at $-80^{\circ} \mathrm{C}$. To relax DNA supercoiling levels and induce biofilm formation, strains were grown in the presence of $10 \mu \mathrm{g} / \mathrm{ml}$ novobiocin as previously described $[27,30]$.

\section{Optical biofilm inhibitor screening}

Bacterial overnight cultures of NCTC11168 (Induced with $10 \mu \mathrm{g} / \mathrm{mL}$ novobiocin) were equalised to an OD600 of 0.1 in $\mathrm{MH}$ broth (supplemented with $10 \mu \mathrm{g} / \mathrm{mL}$ novobiocin) and $200 \mu \mathrm{L}$ were seeded into all of the wells of a CellCarrier Ultra optical 96-well plate (PerkinElmer). The cultures were then incubated for $72 \mathrm{~h}$ at $37^{\circ} \mathrm{C}$ aerobically $\left(21 \% \mathrm{O}_{2}\right)$ to induce adherent biofilm formation. Each row of the 96-well plate was selected for addition of an inhibitor to be tested for its effect on biofilm integrity. After $72 \mathrm{~h}$ incubation, the adherent biofilms in all 96 wells were washed once with PBS in aseptic conditions followed by addition of $100 \mu \mathrm{L} \mathrm{MH}$ broth into all wells except for the first well of each row. For each inhibitor, $200 \mu \mathrm{L}$ of the highest concentration was dissolved in $\mathrm{MH}$ broth $(+10 \mu \mathrm{g} / \mathrm{mL}$ novobiocin $)$ and was added into the first well of each row. The inhibitors used in this study and their highest concentrations were DNAseI (25 U/mL, Sigma), sodium (meta) periodate (4 $\mathrm{mg} / \mathrm{mL}$, Sigma), proteinase K $(20 \mathrm{mg} / \mathrm{mL}$, Promega), trypsin $\left(0.05 \%\right.$, Gibco), $\mathrm{H}_{2} \mathrm{O}_{2}$ (30\% pure, Sigma) and sodium deoxycholate (10\%, Sigma). Using a multiwell pipettor, $100 \mu \mathrm{L}$ of the medium in the first well of each row was diluted in a 1:2 serial dilution along the row to a final volume of $200 \mu \mathrm{L}$. The adherent biofilms were then incubated at $37^{\circ} \mathrm{C}$ for $1 \mathrm{~h}$ in the presence of inhibitors. In order to remove non-adherent bacteria, the wells were then washed 3 times with PBS (Sigma). The metabolically active bacteria were then stained for 30 mins with PBS containing $40 \mu \mathrm{g} / \mathrm{mL}$ 5-TAMRA-SE (5-Carboxytetramethylrhodamine, Succinimidyl Ester, single isomer, ThermoFisher Scientific) viability stain followed by 3 washes with PBS $[28,29]$. This rhodamine-based dye is cell permeant and upon entering the cytosol is enzymatically crosslinked by bacterial esterases, which prevents the rhodamine dye from escaping the intact bacterial cell, providing a bright indicator of living bacteria. Counterstaining of dead bacteria and extracellular DNA structures was subsequently carried out by staining the wells with PBS containing $10 \mu \mathrm{g} / \mathrm{mL}$ SytoX green dye for a further $30 \mathrm{~min}$, followed by 3 additional PBS washing steps [31]. Upon staining with TAMRA/SytoX, the wells were washed with PBS a further 3 times followed by fixing with $4 \%$ PFA for $10 \mathrm{~min}$. Automated confocal microscopy was carried out using an Opera Phenix (PerkinElmer) high-content screening microscope using a $5 x / 0.16 \mathrm{NA}$ air objective. Images were acquired for channels using laser channels $561 \mathrm{~nm}$ (TAMR A) and $488 \mathrm{~nm}$ (SytoX). Images within all experiments were acquired with the same illumination and detection settings.

\section{Automated image analysis of adherent biofilm}

A high-throughput image analysis approach for quantification of TAMRA and SytoX intensity and biofilm area was developed using the Columbus image data and analysis system v.2.8.2 (PerkinElmer). The building blocks involved in the analysis pipeline are described in supplementary Table 1 . The output of the Columbus analysis resulted in a .csv file containing information of quantification of the mean biofilm area $\left(\mathrm{px}^{2}\right)$ and intensity (AU) for each channel.

\section{Data processing and representation}

Quantified adherent biofilm area $\left(\mathrm{px}^{2}\right)$ and intensity (AU) for each inhibited biofilm were normalized to the mean uninhibited biofilm. Data are representative of three biological replicates per condition. Error bars represent the standard deviation for each sample condition. All graphs and statistical analysis were carried out in GraphPad Prism v.6.01 (GraphPad Software). Heatmaps of mean biofilm intensities and area in Fig. 1 were generated using the Python data visualization library 'Seaborn', based on matplotlib.

\section{Results}

Developing an inhibitor screening approach to elucidate the structural composition of induced aerobic biofilm

To allow for a more systematic approach to analyse adherent biofilm formation for larger sample sizes and more conditions, a high-content screening approach was developed as a modification to the biofilm analysis pipeline we developed previously [27]. Optical 96-well plates in which adherent biofilm had formed would proceed to be imaged using an Opera Phenix (PerkinElmer) high- 


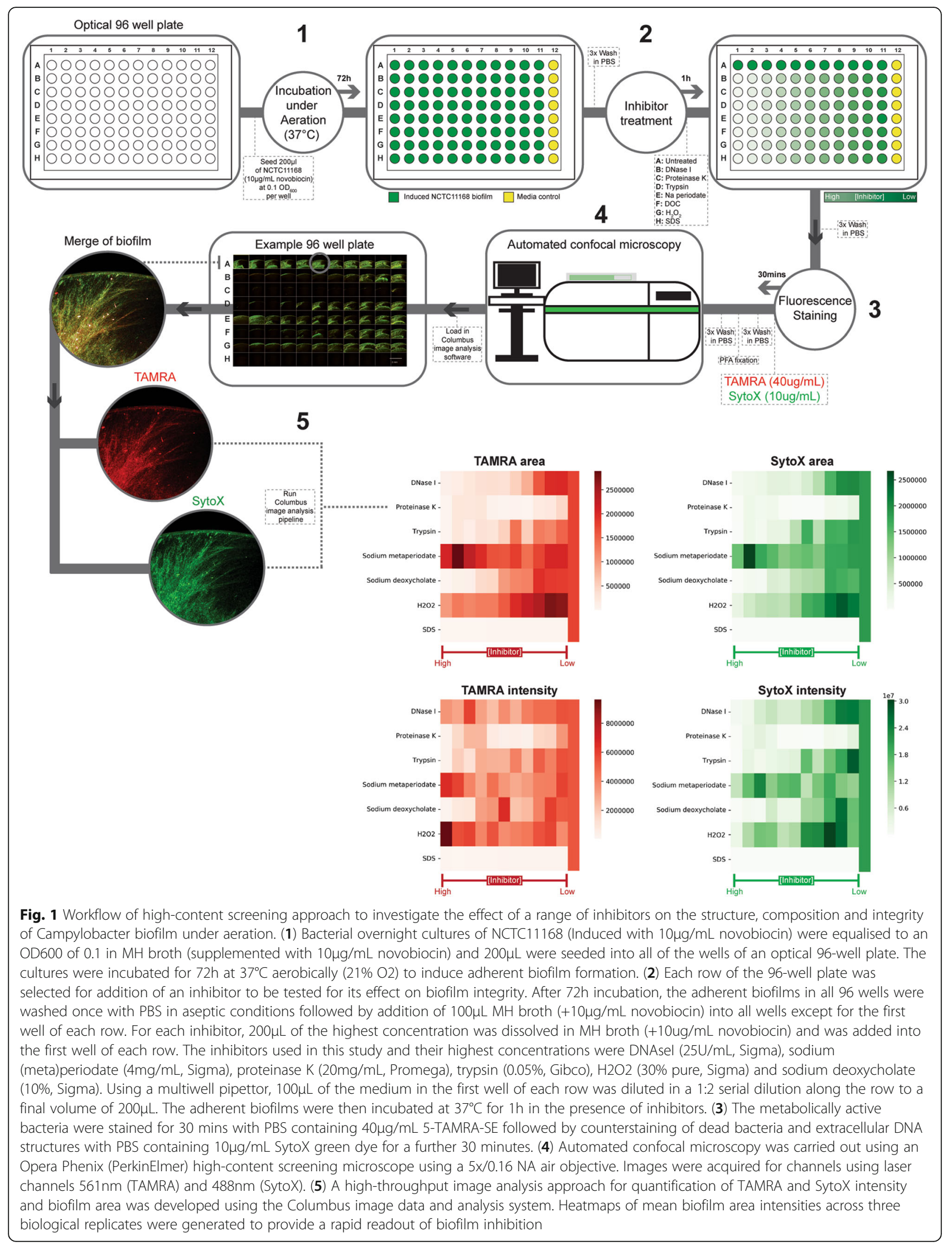


content screening microscope using a 5x/0.16 NA air objective, to allow for a rapid acquisition of confocal images while minimizing the influence of user bias or error during image acquisition. In each 96-well plate, the adherent NCTC11168 biofilm phenotype was induced in 88 wells. Taking into account a positive control row of uninhibited biofilm and a column of $\mathrm{MH}$ broth blank, this allowed for the screening of seven biofilm inhibitors at 11 concentrations.

To gain insight into the molecular composition of the inducible NCTC11168 biofilm after $72 \mathrm{~h}$ incubation in high oxygen conditions, the structural integrity of the EPS component of the adherent biofilm was then challenged with the structural inhibitors chosen to disrupt eDNA (DNase I), EPS carbohydrate (Sodium (meta) periodate) and protein integrity (proteinase $\mathrm{k}$, trypsin). Two biologically relevant compounds were also screened to represent intestinal bile salt stress (sodium deoxycholate), oxidative stress $\left(\mathrm{H}_{2} \mathrm{O}_{2}\right)$. A detergent (sodium dodecyl sulphate) was selected as a positive control. A 1:2 dilution ratio was carried out to serially dilute the inhibitors in each well. Upon exposure to the inhibitors for $1 \mathrm{~h}$, metabolically active biofilm was labelled with $5{ }^{\prime}$-TAMRA-SE and dead cells and eDNA labelled with the nucleic acid dye SytoX. As the workflow in Fig. 1 demonstrates, rapid automated image acquisition was carried out using a PerkinElmer high-content screening confocal microscope. The top of the wells were imaged for each condition as this appeared to be the area in which uninhibited was biofilm consistently present during the PBS washing and aspirating steps. Image acquisition was carried out for TAMRA $(568 \mathrm{~nm})$ and SytoX $(488 \mathrm{~nm})$ channels. For this pilot screen, this resulted in 576,16-bit TIFF images, each averaging 8.9 MB in size, being produced.

Given the large data set, an automated analysis pipeline (Supplementary Table 1) was constructed using the Columbus (PerkinElmer) high-content image analysis software. Adherent biofilms were segmented automatically using a population-based thresholding of the TAMRA and SytoX signal. The fluorescence signal was then thresholded to exclude objects smaller than $4 \mu \mathrm{m}^{2}$ and the area in $\mathrm{px}^{2}$ and intensity (AU) of the biofilm quantified. Using the Seaborn matplotlib python package, a heatmap of the data was rapidly visualized to obtain immediate information about the effect of inhibitors on biofilm integrity (Fig. 1). For quantification of the area and intensity of inhibited TAMRA-stained and SytoX-stained biofilms, each biofilm was normalized to the mean of 11 uninhibited biofilms per replicate, with 1.0 representing an 'uninhibited' biofilm phenotype.

\section{The effect of DNase I and sodium metaperiodate on an induced aerobic biofilm}

As Fig. 2a highlights, as the concentration of DNase I increase there was a dramatic loss of biofilm area with a visible change in eDNA structure (SytoX, green) observable at the low concentration of $0.025 \mathrm{U} / \mathrm{mL}$, which supports our previous observations of the dense eDNA matrix observed [27]. Interestingly, metabolically active bacteria (TAMRA) intensity within the biofilm remained structurally intact at higher concentration even after dissolution of the eDNA matrix, indicating that despite destruction of the biofilm ultrastructure, a significant proportion of bacteria remained adherent and viable.

Sodium (meta) periodate caused minimal observable disruption to the area of TAMRA or SytoX biofilm, even at concentrations as high as $4 \mathrm{mg} / \mathrm{mL}$. Interestingly, at high concentrations of sodium (meta) periodate, the structure of the biofilm appeared to have changed with a visibly less dense matrix (Fig. 2b). This was further supported by a decrease in SytoX positive biofilm intensity when exposed to $4 \mathrm{mg} / \mathrm{mL}$ sodium (meta) periodate.

\section{The effect of proteases on an induced aerobic biofilm}

Proteinase $\mathrm{K}$ and trypsin, which are both serine proteases were very effective at disrupting the integrity of the biofilm (Fig. 3a + b). Proteinase $\mathrm{K}$ was able to dramatically disrupt the inducible biofilm area at concentrations as low as $0.0195 \mathrm{mg} / \mathrm{mL}$ and remove the entire metabolically active NCTC11168 TAMRA intensity signal at $0.078 \mathrm{mg} / \mathrm{mL}$. Trypsin, which was screened at lower concentrations than proteinase $\mathrm{K}$, followed a very similar profile of disruption of SytoX/TAMRA biofilm area and intensity. At concentrations of $0.001 \%$ the trends of disruption of the two biofilm stains differed with the eDNA/DNA stained biofilm being totally disrupted while a proportion of the metabolically active (TAMRA) cells remained adherent (Fig. 3a).

\section{The effect of environmental stressors sodium deoxycholate and hydrogen peroxide on induced aerobic biofilm integrity}

The ability of a viable biofilm to survive and maintain structural integrity under two environmental stressors that would be encountered during $C$. jejuni pathogenesis from the farm to the jejunum was next determined. Firstly, the antimicrobial bile salt sodium deoxycholate, which is encountered in the intestine was selected [32, 33]. Secondly, $\mathrm{H}_{2} \mathrm{O}_{2}$ which promotes the oxidative stress response already observed during aeration, in the phagosome and also may play a role in the intestinal epithelium in mucosal defence against microbial pathogens [34]. The detergent sodium dodecyl sulphate (SDS) was also used as a positive control for total disruption of the biofilm.

NCTC11168-induced biofilm structure was significantly disrupted by concentrations of sodium deoxycholate at concentrations of $0.3125 \% \mathrm{w} / \mathrm{v}$. However, a visible 
A

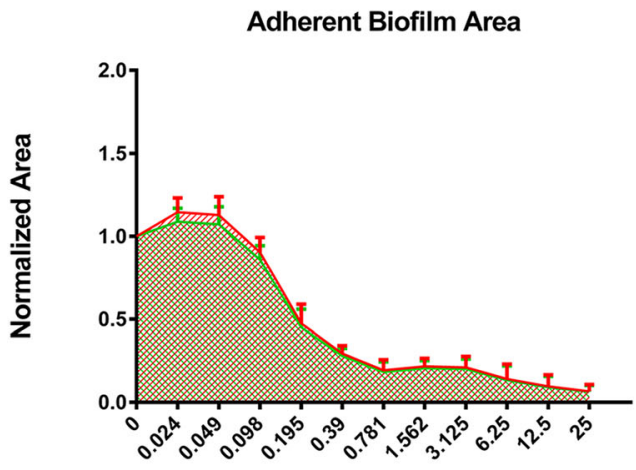

DNase I Concentration (U/mL)

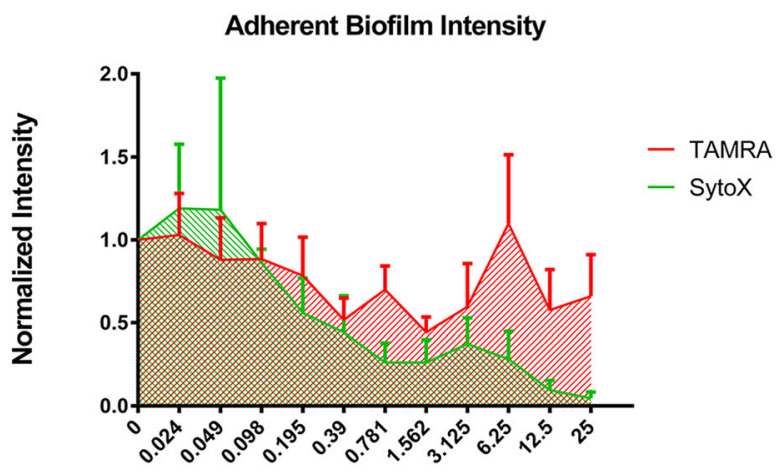

DNase I Concentration $(\mathrm{U} / \mathrm{mL})$
$0 \mathrm{U} / \mathrm{mL}$

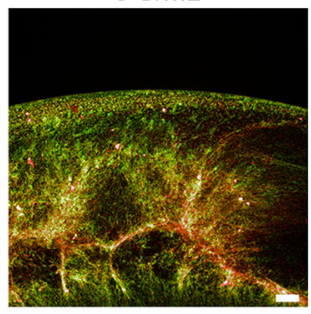

$0.024 \mathrm{U} / \mathrm{mL}$

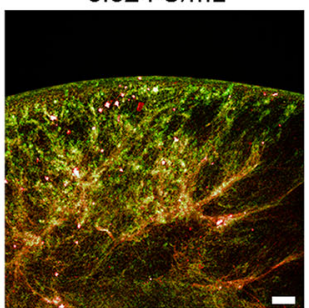

$0.195 \mathrm{U} / \mathrm{mL}$

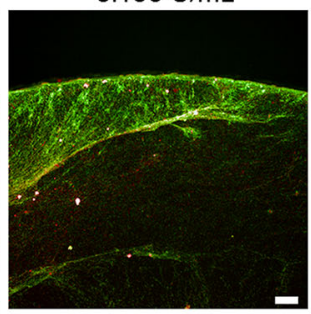

$6.25 \mathrm{U} / \mathrm{mL}$

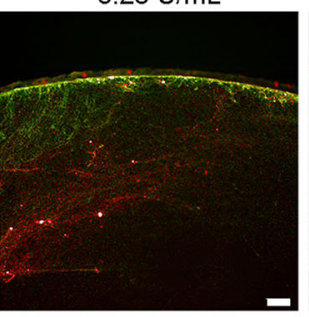

$25 \mathrm{U} / \mathrm{mL}$

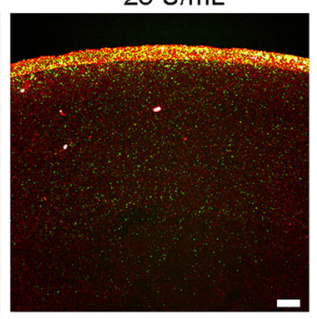

B

Adherent Biofilm Area

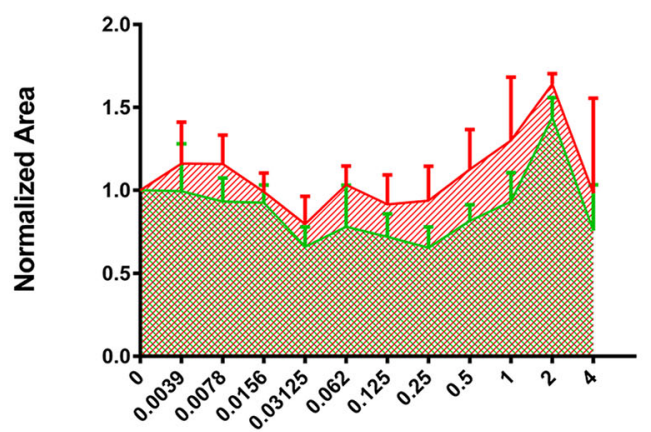

Sodium metaperiodate concentration $(\mathrm{mg} / \mathrm{mL})$
Adherent Biofilm Intensity

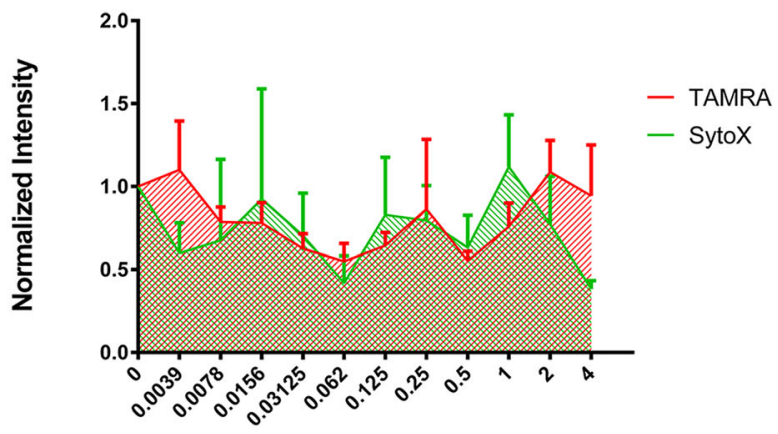

Sodium metaperiodate concentration $(\mathrm{mg} / \mathrm{mL})$
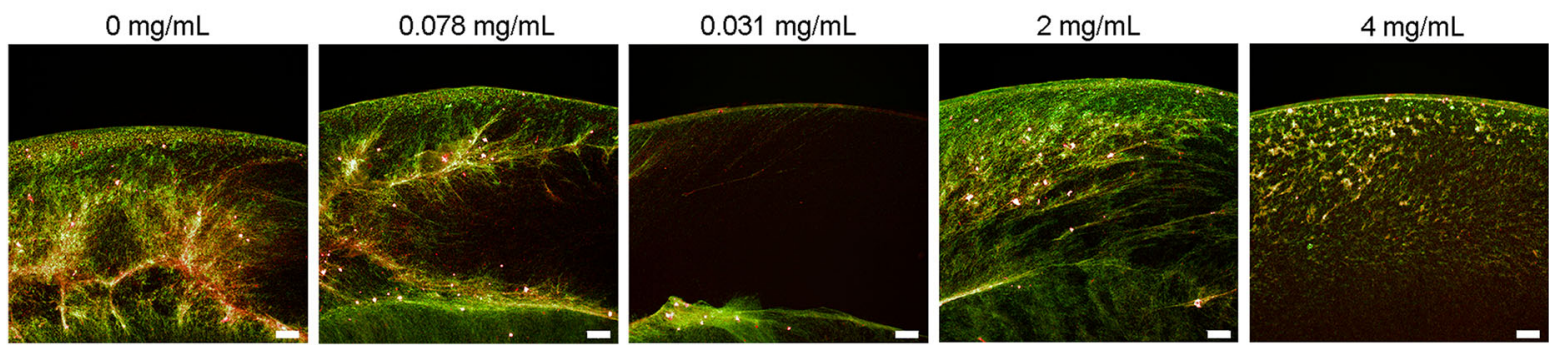

Fig. 2 (A) Investigating the ability of DNase I to disrupt adherent biofilm of NCTC11168 induced with 10 $\mathrm{\mu g} / \mathrm{mL}$ novobiocin, under aeration. Quantification of TAMRA and SyoX labelled adherent biofilm area (px2) (Left) and intensity (AU) (Right), normalised to uninhibited biofilm. (Bottom) Images of TAMRA labelled (red) and SytoX (green) labelled biofilm post-incubation with DNase I. (B) Investigating the ability of sodium (meta) periodate to disrupt adherent biofilm of NCTC11168 induced with 10 $\mathrm{\mu g} / \mathrm{mL}$ novobiocin, under aeration. Quantification of TAMRA and SyoX labelled adherent biofilm area (px2) (Left) and intensity (AU) (Right), normalised to uninhibited biofilm. (Bottom) Images of TAMRA labelled (red) and SytoX (green) labelled biofilm post-incubation with sodium periodate. Data shown is mean of three replicates per condition $+/-\mathrm{SD}, \mathrm{n}=3$. Scale bar $=100 \mu m$ 
A

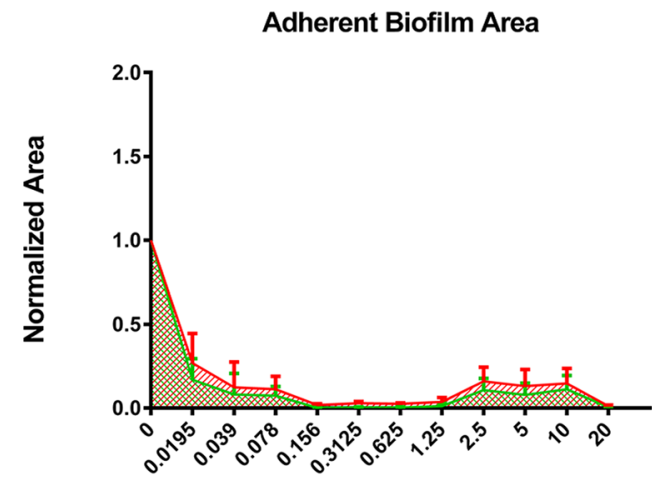

Proteinase $\mathrm{K}$ concentration $(\mathrm{mg} / \mathrm{mL})$

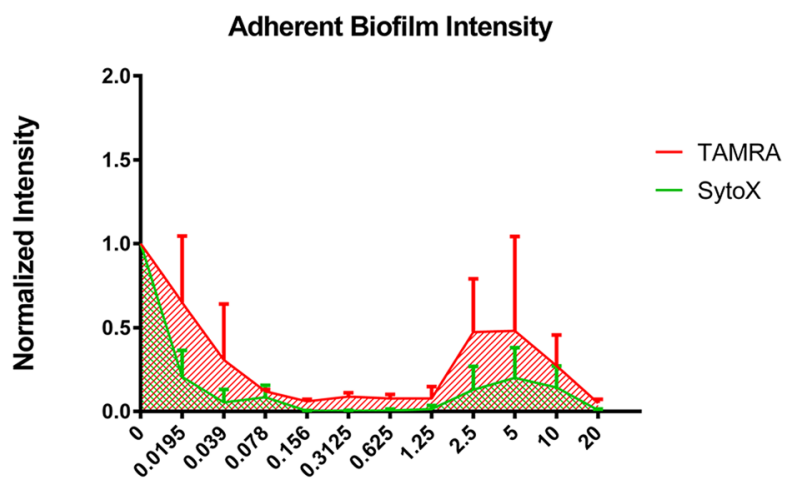

Proteinase $\mathrm{K}$ concentration $(\mathrm{mg} / \mathrm{mL}$ )
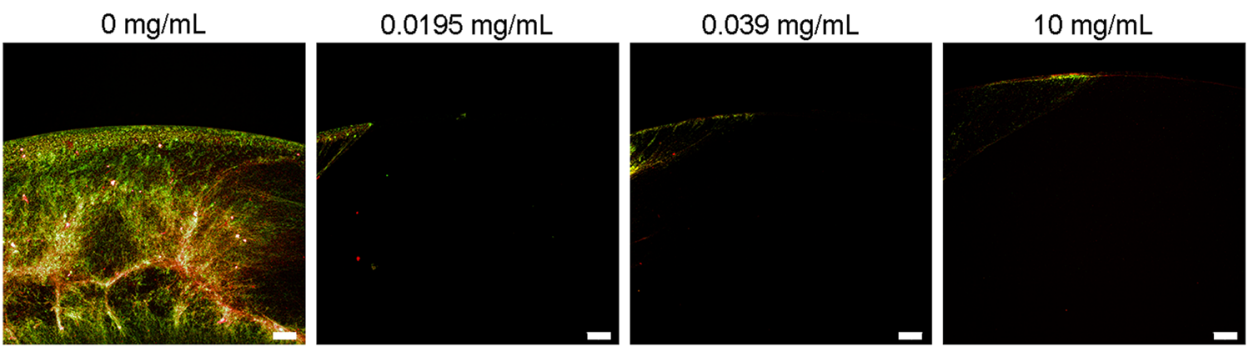

$20 \mathrm{mg} / \mathrm{mL}$

B

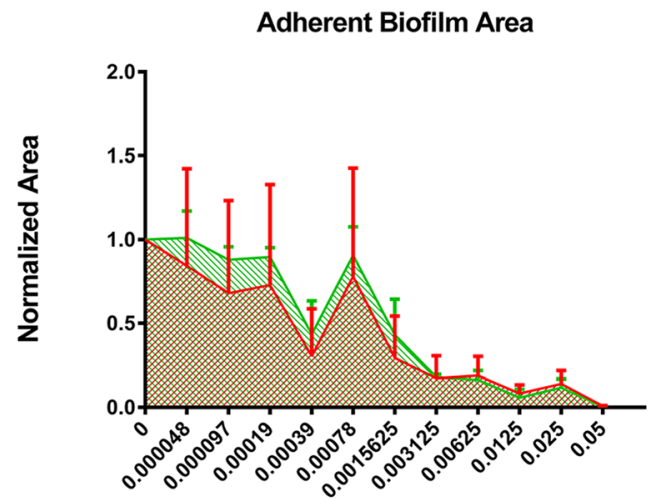

Trypsin concentration (\%)

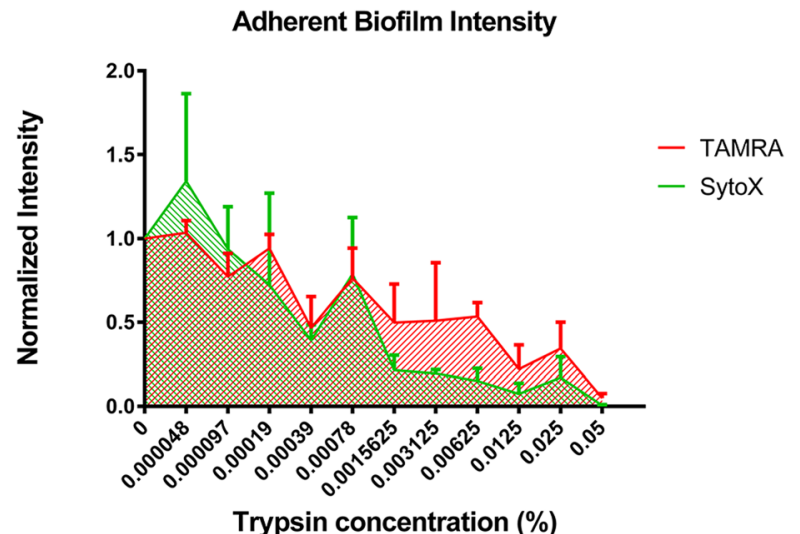

$0 \%$

$0.000097 \%$

$0.00019 \%$

$0.025 \%$

$0.05 \%$
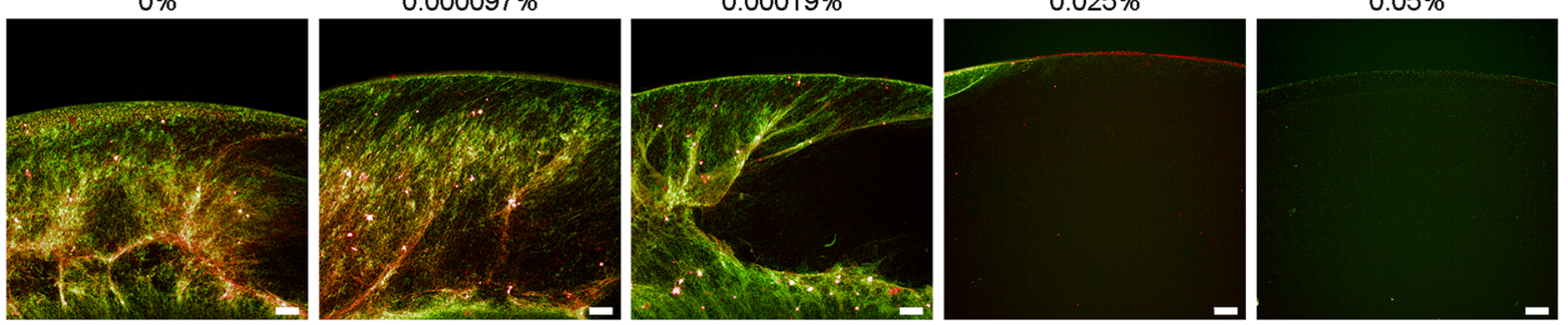

Fig. 3 (A) Investigating the ability of proteinase K to disrupt adherent biofilm of NCTC11168 induced with 10 $\mathrm{\mu g} / \mathrm{mL}$ novobiocin, under aeration. Quantification of TAMRA and SyoX labelled adherent biofilm area (px2) (Left) and intensity (AU) (Right), normalised to uninhibited biofilm. (Bottom) Images of TAMRA labelled (red) and SytoX (green) labelled biofilm post-incubation with Proteinase k. (B) Investigating the ability of

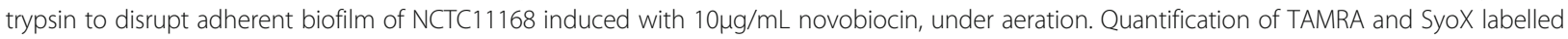
adherent biofilm area (px2) (Left) and intensity (AU) (Right), normalised to uninhibited biofilm. (Bottom) Images of TAMRA labelled (red) and SytoX (green) labelled biofilm post-incubation with trypsin. Data shown is mean of three replicates per condition $+/-\mathrm{SD}, \mathrm{n}=3$. Scale bar $=100 \mu \mathrm{m}$ 
trend of disruption was evident at $0.078 \% \mathrm{w} / \mathrm{v}$ for both TAMRA and SytoX positive biofilm (Fig. 4a). This was interesting as the physiologically relevant concentration of sodium deoxycholate in the human intestine is $0.1 \%$ $\mathrm{w} / \mathrm{v}$ [19].

As this NCTC11168 biofilm was induced specifically under aeration, the NCTC11168 biofilm was highly resistant to $1 \mathrm{~h}$ exposure to $\mathrm{H}_{2} \mathrm{O}_{2}$. At low concentrations of $\mathrm{H}_{2} \mathrm{O}_{2}, 0.029-0.117 \%$ for SytoX, and $0.029-0.234 \%$ for TAMRA, a significant increase in biofilm area compared to the uninhibited biofilm was observed.

Disruption of both TAMRA positive and SytoX positive biofilm area was dramatic after $1 \mathrm{~h}$ incubation with $1.8765 \% \mathrm{H}_{2} \mathrm{O}_{2}$. However, clusters of metabolically active cells were still observed to be attached at concentration of $7.5 \%$ or higher (Fig. $4 \mathrm{~b}$ ) which was evidenced by the relative lack of disruption of TAMRA signal intensity at high concentrations of $\mathrm{H}_{2} \mathrm{O}_{2}$. Treatment of the induced biofilm with the detergent sodium dodecyl sulphate (SDS) resulted in total disruption of both adherent bacteria and eDNA matrix components at concentrations as low as $0.029 \%$. This clearly Indicates the power of surfactants at removal of $C$. jejuni adherent biofilm from an abiotic surface (Supplementary Figure 1).

\section{Discussion}

A significant challenge in the field of Campylobacter biofilm research has been the low-throughput of traditional imaging based methodological approaches and biofilm heterogeneity amongst strains in which much of the work has been done to investigate the core EPS composition. The uniformity of adherent biofilm produced by the induced aerobic biofilm phenotype shown in our previous work [27] offered a unique opportunity to robustly screen large numbers of homogenous Campylobacter biofilms using a variety of relevant inhibitors. Biofilm formation under aeration was of particular interest, as high oxygen levels are the most prominent environmental stressor experienced by $C$. jejuni residing on chicken carcasses, during the transmission to humans. Indeed, high levels of available oxygen are capable of relaxing negative supercoiling of bacterial DNA. Bacterial pathogens such as S. typhimurium take advantage of supercoiling sensitive regulators of gene expression to express cohorts of genes conferring the tools necessary to survive in oxidative stress inducing environments such as intracellular environment of macrophages [35].

An advantage of the development of the high-content screening approach described here to assess adherent biofilm formation is that it was possible to robustly screen for inhibitors of the biofilm induced by relaxation of DNA supercoiling under aeration. A significant advantage of this approach is that it allowed for a large number of positive controls, 33 uninhibited biofilms, to be assessed. This therefore provided a robust quantification of the baseline size and intensity of Campylobacter biofilm. The use of a fluorescent labelling system allowed for the quantification of the effect of biofilm size (area) and density (intensity) for the metabolically active living bacterial population (TAMRA) as well as the largely eDNA EPS component of the biofilm (SytoX).

A pilot screen was conducted to broadly investigate the EPS structural composition of the induced biofilm of NCTC11168 by treatment with increasing concentrations of DNase I (degradation of eDNA), sodium (meta) periodate (oxidisation/ disruption of carbohydrate), proteinase $\mathrm{K}$ and trypsin (serine protease mediated protein cleavage). As expected, degradation of eDNA led to efficient disruption of the induced biofilm between the concentrations of $0.39 \mathrm{U} / \mathrm{mL}$ to $25 \mathrm{U} / \mathrm{mL}$. This was in agreement with the observations of Brown and colleagues [24]. However, they found that biofilm inhibition was observed at concentrations of DNase I of $0.01 \mathrm{U} / \mathrm{mL}$ which may indicate that an increase in eDNA matrix amount and density plays a significant role in this inducible biofilm phenotype. Interestingly, when incubated with $0.39 \mathrm{U} / \mathrm{mL}$ DNase I, a large proportion of biofilm remained adherent to the surface, while SytoX staining of DNA was largely abolished (Fig. 2a). A carbohydrate moiety did not appear to be present within the EPS of the induced biofilm as it was insensitive to treatment with sodium (meta) periodate, maintaining biofilm integrity and viability throughout treatment, which was in agreement with the observations of Melo and colleagues (2017) [26]. Interestingly, at high concentrations (4 mg/ $\mathrm{mL}$ ), sodium (meta) periodate, the 3D ultrastructure of the adherent biofilm changed in appearance (Fig. 2b). This was correlated with a sharp decline in the SytoX positive biofilm moiety. It is likely that disruption of surface glycans and possibly capsular protein glycosylation was to blame for this change in structure, however further work would have to be carried out to confirm this.

In agreement with the observations of the key role of bacterial adhesion in the relaxation of DNA supercoiling induced biofilm, both proteinase $\mathrm{K}(\geq 19.5 \mu \mathrm{g} / \mathrm{mL})$ and trypsin $(\geq 0.00039 \%)$ were highly efficient at disrupting the induced biofilm, likely through disruption of cellcell/cell-surface adhesion mediated by the flagella and $\mathrm{OM}$ adhesins. These observations were also in line with the findings of Melo and colleagues (2017), who found that proteinase $\mathrm{K}$ was highly efficient at disrupting $C$. jejuni biofilm at $1 \mathrm{mg} / \mathrm{mL}$. They suggested that costs of production are the main limiting factor in the use of proteinase $\mathrm{K}$ in biotreatment of $C$. jejuni biofilms in an agricultural setting, however the findings in this study suggest that proteinase $\mathrm{K}$ is highly efficient as clearing $C$. jejuni biofilm at very low treatment doses. Taken together, these findings are largely in agreement with the 
A

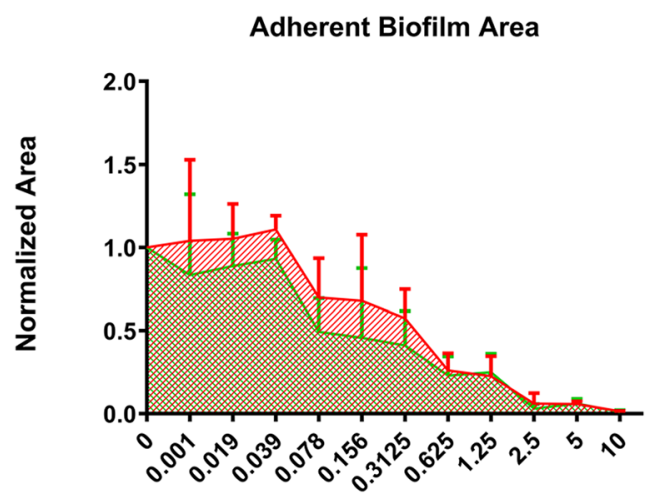

DOC concentration (\%)

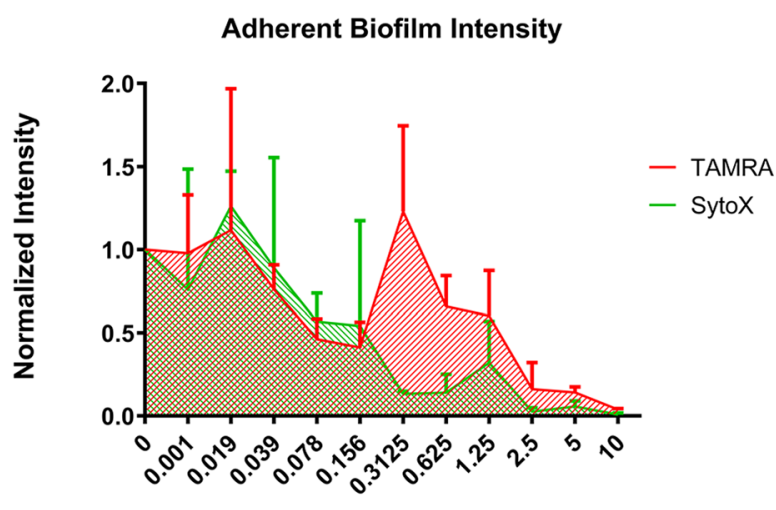

DOC concentration (\%)
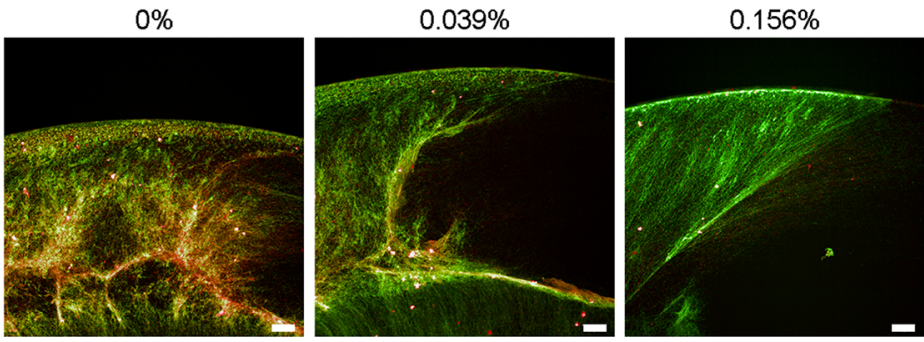

$0.3125 \%$
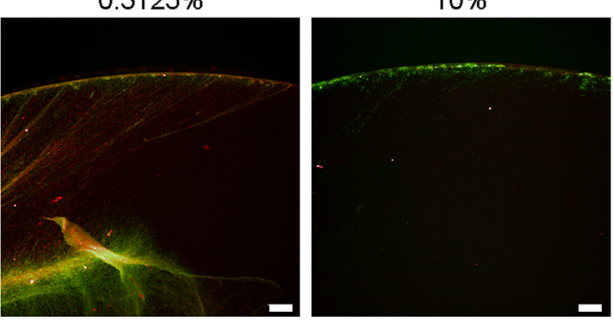

B

Adherent Biofilm Area

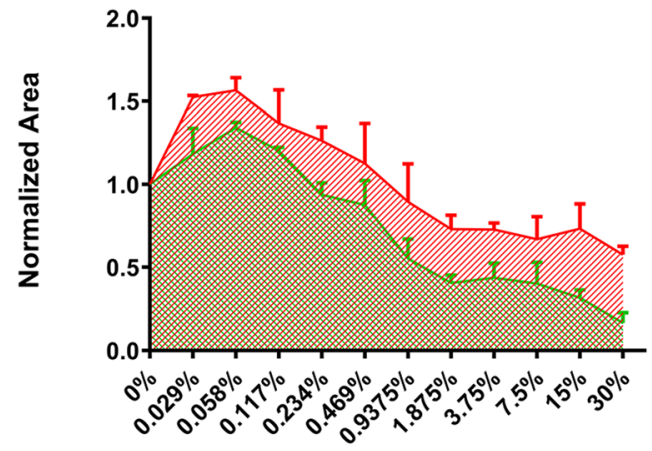

$\mathrm{H}_{2} \mathrm{O}_{2}$ concentration (\%)

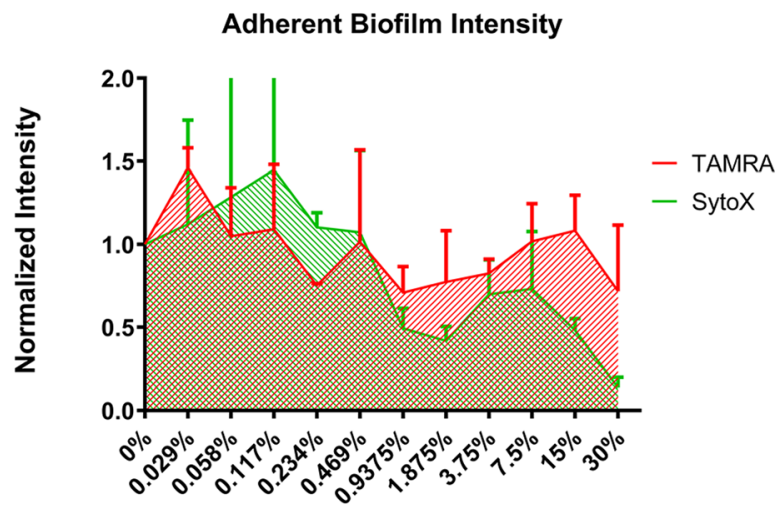

$\mathrm{H}_{2} \mathrm{O}_{2}$ concentration (\%)
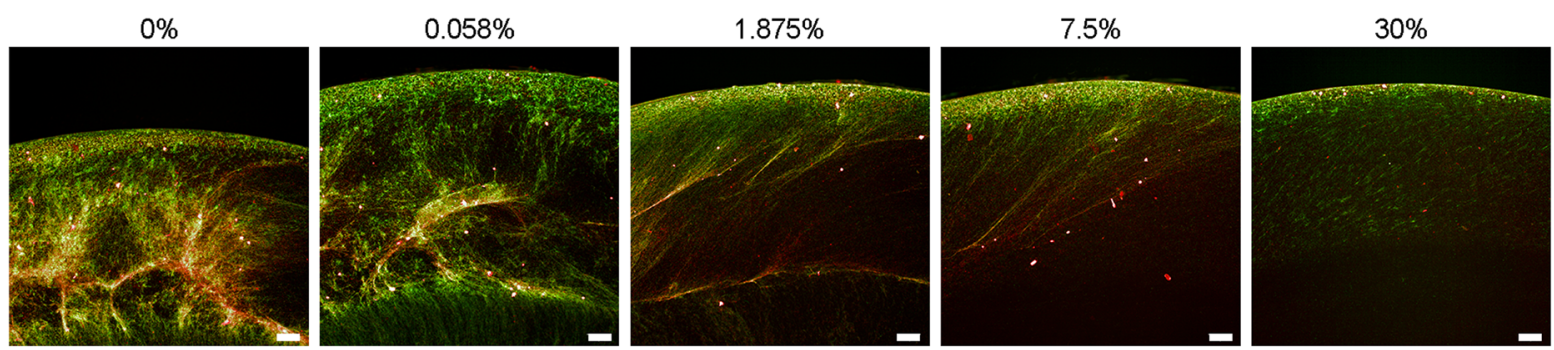

Fig. 4 (A) Investigating the ability of sodium deoxycholate (DOC) to disrupt adherent biofilm of NCTC11168 induced with 10 $\mu \mathrm{g} / \mathrm{mL}$ novobiocin, under aeration. Quantification of TAMRA and SyoX labelled adherent biofilm area (px2) (Left) and intensity (AU) (Right), normalised to uninhibited biofilm. (Bottom) Images of TAMRA labelled (red) and SytoX (green) labelled biofilm post-incubation with DOC. (B) Investigating the ability of $\mathrm{H} 2 \mathrm{O} 2$ to disrupt adherent biofilm of NCTC11168 induced with $10 \mu \mathrm{g} / \mathrm{mL}$ novobiocin, under aeration. Quantification of TAMRA and SyoX labelled adherent biofilm area (px2) (Left) and intensity (AU) (Right), normalised to uninhibited biofilm. (Bottom) Images of TAMRA labelled (red) and SytoX (green) labelled biofilm post-incubation with H2O2. Data shown is mean of three replicates per condition $+/-\mathrm{SD}, \mathrm{n}=3$. Scale bar $=100 \mu \mathrm{m}$ 
current literature, further supporting that this supercoiling induced biofilm is structurally representative of biofilms naturally formed by $C$. jejuni.

Three inhibitors representing 'environmental stressors' were screened for their ability to disrupt the novobiocininduced biofilm phenotype of NCTC11168, the bile salt sodium deoxycholate (DOC), $\mathrm{H}_{2} \mathrm{O}_{2}$ and sodium dodecyl sulphate (SDS). Firstly, DOC has been shown to induce a highly secretory invasive phenotype in C. jejuni [19]. DOC has also been shown to enhance biofilm formation with incubation of strain 81-176 in the presence of 0.05\% DOC promoting adherent biofilm formation, autolysis and eDNA release [1]. Interestingly, no increase in adherent biofilm formation at 'subinhibitory' concentrations of DOC treatment was observed (Fig. 4a). At concentrations of $0.3125 \%$ DOC and above, disruption of the adherent biofilm occurred. It is possible that the increase observed by Svensson and colleagues (2014) occurs specifically when incubated microaerobically, or there is a possibility that the novobiocin-induced phenotype represents the 'maximum' biofilm possible by this number of bacteria, therefore being insensitive to the regulatory triggers of DOC. Interestingly, at concentrations of $0.3125-1.25 \%$ DOC treatment, remediation of the inhibition of TAMRA positive biofilm intensity was observed. Indicating that under these conditions there was a significant proportion of the metabolically active cells within the population remaining adherent to the well.

Treatment with $\mathrm{H}_{2} \mathrm{O}_{2}$ was utilised to investigate maintenance of biofilm integrity in oxidative stress coupled with aerobic stress. Interestingly, the novobiocin-induced biofilm was uninhibited until treatment with $0.9376 \%$ $\mathrm{H}_{2} \mathrm{O}_{2}$ or higher (Fig. 4b). It was notable that at very low levels of dissolved $\mathrm{H}_{2} \mathrm{O}_{2}$ (0.029 and $0.058 \%$ ), there was an increase in viable biofilm in addition to the novobiocininduced biofilm. This could be explained by the further relaxation of DNA supercoiling homeostasis by ROS at subinhibitory levels. This observation supports the hypothesis that this high oxygen abundance acts as a major environmental driving factor to promote global upregulation of genes involved in biofilm formation, invasion and oxidative stress response in $C$. jejuni. The observation that viable (red) bacteria were present after incubation in levels of $7.5 \% \mathrm{H}_{2} \mathrm{O}_{2}$ or greater (up to $30 \%$ ) appear to be higher than the $\mathrm{H}_{2} \mathrm{O}_{2}$ tolerance levels reported for $C$. jejuni biofilm in the literature [36]. This indicates that this viable biofilm would not only provide benefits to host defence reactive oxygen species but also promote transmission via survival in the high oxygen environment of the supermarket. This could also confer advantages to survival of $\mathrm{H}_{2} \mathrm{O}_{2}$-mediated cleaning using $10-30 \% \mathrm{H}_{2} \mathrm{O}_{2}$ resulting in less efficient killing of the C. jejuni. Interestingly, the $\triangle p e r R$ mutant is capable of surviving in far higher levels of $\mathrm{H}_{2} \mathrm{O}_{2}$ than WT C. jejuni, due to the derepression of oxidative stress response genes controlled by the PerR regulon [36, 37]. It is reasonable to suggest that relaxation of DNA supercoiling, which is induced by presence of oxygen, can upregulate the secretion of oxidative stress response genes which in conjunction with modification of the flagella and increasing OMP adhesins, promote biofilm formation in aerobic conditions.

In the past, high-content screening approaches have predominantly been utilised in antimicrobial drug screening when implemented in the context of microbial pathogenesis. In this work we present a novel imagingbased biofilm screening platform. This methodology could provide significant advantages in the rapid testing of the ability of large numbers of $C$. jejuni strains to form biofilm in a variety of environmental contexts, as well as to dissect the molecular mechanisms at play during C. jejuni biofilm formation. This approach also offers the opportunity to be easily adapted to phenotypically screen and characterise adherent biofilm of large numbers of fresh bacterial isolates of a variety of bacterial pathogens. In the modern era of big 'omic' data in relation to genomic, transcriptomics and proteomics, approaches such as the one described here offer an opportunity to study biofilm formation by pathogens on a much larger scale.

\section{Supplementary Information}

The online version contains supplementary material available at https://doi. org/10.1186/s12866-020-02062-5.

Additional file 1. Automated image analysis pipeline as executed using the PerkinElmer Columbus image analysis suite. Figure 1. (A)

Investigating the ability of sodium dodecyl sulphate (SDS) to to disrupt adherent biofilm of NCTC11168 induced with $10 \mu \mathrm{g} / \mathrm{mL}$ novobiocin, under aeration. Quantification of TAMRA and SyoX labelled adherent biofilm area (px2) (Left) and intensity (AU) (Right), normalised to uninhibited biofilm. (Bottom) Images of TAMRA labelled (red) and SytoX (green) labelled biofilm post-incubation with SDS. Data shown is mean of three replicates per condition $+/-\mathrm{SD}, \mathrm{n}=3$. Scale bar $=100 \mu \mathrm{m}$.

Authors' contributions

All experiments were carried out by M.W. and the project was co-supervised by J.S. and T.OC. All authors read and approved the final manuscript.

\section{Competing interests}

The authors declare no competing interests.

\section{Author details}

${ }^{1}$ School of Biomolecular and Biomedical Science, University College Dublin, Belfield, Dublin 4, Ireland. ${ }^{2}$ School of Biology and Environmental Science, University College Dublin, Belfield, Dublin 4, Ireland.

Received: 14 September 2020 Accepted: 23 November 2020

Published online: 04 January 2021

\section{References}

1. Svensson SL, Pryjma M, Gaynor EC. Flagella-mediated adhesion and extracellular DNA release contribute to biofilm formation and stress tolerance of Campylobacter jejuni. PLoS One. 2014;9(8):e106063. 
2. Reeser RJ, Medler RT, Billington SJ, Jost BH, Joens LA. Characterization of campylobacter jejuni biofilms under defined growth conditions. Appl Environ Microbiol. 2007;73(6):1908-13.

3. Reuter M, Mallett A, Pearson BM, Van Vliet AHM. Biofilm formation by campylobacter jejuni is increased under aerobic conditions. Appl Environ Microbiol. 2010;76(7):2122-8.

4. Joshua GWP, Guthrie-Irons C, Karlyshev AV, Wren BW. Biofilm formation in campylobacter jejuni. Microbiology. 2006;152(2):387-96.

5. McLennan MK, et al. Campylobacter jejuni biofilms up-regulated in the absence of the stringent response utilize a calcofluor white-reactive polysaccharide. J Bacteriol. 2008;190(3):1097-107.

6. Candon HL, Allan BJ, Fraley CD, Gaynor EC. Polyphosphate kinase 1 is a pathogenesis determinant in campylobacter jejuni. J Bacteriol. 2007;189(22): 8099-108.

7. Gangaiah D, et al. Polyphosphate kinase 2: A novel determinant of stress responses and pathogenesis in campylobacter jejuni. PLoS One. 2010;5(8): e12142.

8. Kalmokoff M, et al. Proteomic analysis of campylobacter jejuni 11168 biofilms reveals a role for the motility complex in biofilm formation. $J$ Bacteriol. 2006;188(12):4312-20.

9. Svensson SL, et al. The CprS sensor kinase of the zoonotic pathogen campylobacter jejuni influences biofilm formation and is required for optimal chick colonization. Mol Microbiol. 2009;71(1):253-72.

10. Naito $M$, et al. Effects of sequential campylobacter jejuni 81-176 lipooligosaccharide core truncations on biofilm formation, stress survival, and pathogenesis. J Bacteriol. 2010;192(8):2182-92.

11. Lim ES, Kim JS. Role of eptC in biofilm formation by campylobacter jejuni NCTC11168 on polystyrene and glass surfaces. J Microbiol Biotechnol. 2017; 27(9):1609-16.

12. Rathbun KM, Thompson SA. Mutation of PEB4 alters the outer membrane protein profile of campylobacter jejuni. FEMS Microbiol Lett. 2009;300(2): 188-94.

13. Fields JA, Thompson SA. Campylobacter jejuni CsrA mediates oxidative stress responses, biofilm formation, and host cell invasion. J Bacteriol. 2008; 190(9):3411-6

14. Sulaeman S, et al. Enhanced Adhesion of Campylobacter jejuni to Abiotic Surfaces Is Mediated by Membrane Proteins in Oxygen-Enriched Conditions. PLoS One. 2012;7(9):e46402.

15. Theoret JR, et al. The campylobacter jejuni dps homologue is important for in vitro biofilm formation and cecal colonization of poultry and may serve as a protective antigen for vaccination. Clin Vaccine Immunol. 2012;19(9): 1426-31.

16. Poly $F$, et al. Heterogeneity of a Campylobacter jejuni\&lt;/em\&gt; Protein That Is Secreted through the Flagellar Filament. Infect Immun. 2007;75(8): 3859-67.

17. Haddock G, et al. Campylobacter jejuni 81-176 forms distinct microcolonies on in vitro-infected human small intestinal tissue prior to biofilm formation. Microbiology. 2010;156(10):3079-84.

18. MOE KK, et al. The mode of biofilm formation on smooth surfaces by campylobacter jejuni. J Vet Med Sci. 2010;72(4):411-6.

19. Malik-Kale P, Parker CT, Konkel ME. Culture of campylobacter jejuni with sodium deoxycholate induces virulence gene expression. J Bacteriol. 2008; 190(7):2286-97.

20. Brown HL, Reuter M, Salt $L$, Cross KL, Betts RP, van Vliet AHM. Chicken juice enhances surface attachment and biofilm formation of campylobacter jejuni. Appl Environ Microbiol. 2014;80(22):7053-60.

21. Turonova $\mathrm{H}$, et al. Biofilm spatial organization by the emerging pathogen Campylobacter jejuni: comparison between NCTC 11168 and 81-176 strains under microaerobic and oxygen-enriched conditions. Front Microbiol. 2015; 6(July):709.

22. Oh E, Kim JC, Jeon B. Stimulation of biofilm formation by oxidative stress in campylobacter jejuni under aerobic conditions. Virulence. 2016;7(7):846-51.

23. Flemming H-C, Wingender J. The biofilm matrix. Nat Rev Microbiol. 2010; 8(9):623-33.

24. Brown HL, Hanman K, Reuter M, Betts RP, van Vliet AHM. Campylobacter jejuni biofilms contain extracellular DNA and are sensitive to DNase I treatment. Front Microbiol. 2015;6(JUL):1-11.

25. Montanaro L, et al. Extracellular DNA in biofilms. Int J Artif Organs. 2011; 34(9):824-31.

26. Melo RT, et al. Intrinsic and extrinsic aspects on Campylobacter jejuni Biofilms. Front Microbiol. 2017;8:1332.
27. Whelan MVX, et al. Acquisition of fluoroquinolone resistance leads to increased biofilm formation and pathogenicity in Campylobacter jejuni. Sci Rep. 2019;9(1):18216.

28. Mooney A, Byrne C, Clyne M, Johnson-Henry K, Sherman P, Bourke B. Invasion of human epithelial cells by campylobacter upsaliensis. Cell Microbiol. 2003;5(11):835-47.

29. Byrne CM, Clyne M, Bourke B. Campylobacter jejuni adhere to and invade chicken intestinal epithelial cells in vitro. Microbiology. 2007;153(2):561-9.

30. Scanlan $\mathrm{E}$, et al. Relaxation of DNA supercoiling leads to increased invasion of epithelial cells and protein secretion by Campylobacter jejuni. Mol Microbiol. 2017:104(1):92-104.

31. Okshevsky M, Meyer RL. Evaluation of fluorescent stains for visualizing extracellular DNA in biofilms. J Microbiol Methods. 2014;105:102-4.

32. Hofmann AF. THe continuing importance of bile acids in liver and intestinal disease. Arch Intern Med. 1999:159(22):2647-58.

33. Begley M, Sleator RD, Gahan CGM, Hill C. Gastrointestinal persistence and bile tolerance of listeria monocytogenes. Infect Immun. 2005;73(2):894-904.

34. Corcionivoschi $\mathrm{N}$, et al. Mucosal reactive oxygen species decrease virulence by disrupting campylobacter jejuni Phosphotyrosine signaling. Cell Host Microbe. 2012:12(1):47-59.

35. Cameron ADS, et al. Transmission of an oxygen availability signal at the salmonella enterica serovar typhimurium fis promoter. PLoS One. 2013;8(12): $1-12$

36. Handley RA, et al. PerR controls oxidative stress defence and aerotolerance but not motility-associated phenotypes of campylobacter jejuni. Microbiology (United Kingdom). 2015;161(7):1524-36.

37. Palyada K, Sun YQ, Flint A, Butcher J, Naikare H, Stintzi A. Characterization of the oxidative stress stimulon and PerR regulon of campylobacter jejuni. BMC Genomics. 2009;10:481.

\section{Publisher's Note}

Springer Nature remains neutral with regard to jurisdictional claims in published maps and institutional affiliations.
Ready to submit your research? Choose BMC and benefit from:

- fast, convenient online submission

- thorough peer review by experienced researchers in your field

- rapid publication on acceptance

- support for research data, including large and complex data types

- gold Open Access which fosters wider collaboration and increased citations

- maximum visibility for your research: over $100 \mathrm{M}$ website views per year

At $\mathrm{BMC}$, research is always in progress.

Learn more biomedcentral.com/submissions 